\title{
FINITE ELEMENTS FOR MATERIALS WITH STRAIN GRADIENT EFFECTS
}

\author{
JOHN Y. SHU ${ }^{1, *}$, WAYNE E. KING ${ }^{1}$ AND NORMAN A. FLECK ${ }^{2}$ \\ ${ }^{1}$ Chemistry and Materials Science Department, Lawrence Livermore National Laboratory, Livermore, CA 94550, U.S.A. \\ ${ }^{2}$ Cambridge University Engineering Department, Trumpington Street, Cambridge, CB2 1PZ, U.K.
}

\begin{abstract}
SUMMARY
A finite element implementation is reported of the Fleck-Hutchinson phenomenological strain gradient theory. This theory fits within the Toupin-Mindlin framework and deals with first-order strain gradients and the associated work-conjugate higher-order stresses. In conventional displacement-based approaches, the interpolation of displacement requires $C^{1}$-continuity in order to ensure convergence of the finite element procedure for higher-order theories. Mixed-type finite elements are developed herein for the Fleck-Hutchinson theory; these elements use standard $C^{0}$-continuous shape functions and can achieve the same convergence as $C^{1}$ elements. These $C^{0}$ elements use displacements and displacement gradients as nodal degrees of freedom. Kinematic constraints between displacement gradients are enforced via the Lagrange multiplier method. The elements developed all pass a patch test. The resulting finite element scheme is used to solve some representative linear elastic boundary value problems and the comparative accuracy of various types of element is evaluated. Copyright (C) 1999 John Wiley \& Sons, Ltd.
\end{abstract}

KEY WORDS: strain gradient; finite element

\section{INTRODUCTION}

Conventional continuum mechanics theories assume that stress at a material point is a function of 'state' variables, such as strain, at the same point. This local assumption has long been proved to be adequate when the wavelength of a deformation field is much larger than the dominant micro-structural length scale of the material. However, when the two length scales are comparable, the assumption is questionable as the material behaviour at a point is influenced by the deformation of neighbouring points. Starting from the pioneering Cosserat couple stress theory, ${ }^{1}$ various non-local or strain gradient continuum theories have been proposed. In the full Cosserat theory, ${ }^{1}$ an independent rotation quantity $\boldsymbol{\theta}$ is defined in addition to the material displacement $\mathbf{u}$; couple stresses (bending moment per unit area) are introduced as the work conjugate to the micro-curvature (that is, the spatial gradient of $\boldsymbol{\theta}$ ). Later, Toupin ${ }^{2}$ and Mindlin ${ }^{3}$ proposed a more general theory which includes not only micro-curvature, but also gradients of normal strain. Both

\footnotetext{
* Correspondence to: John Y. Shu, Chemistry and Materials Science Department, Lawrence Livermore National Laboratory, Livermore, CA 94550, U.S.A. E-mail: jyshu@1lnl.gov

Contract/grant sponsor: U.S. Department of Energy

Contract/grant sponsor: Lawrence Livermore National Laboratory; Contract/grant number: W-7405-Eng-48
}

CCC 0029-5981/99/030373-19\$17.50

Copyright (C) 1999 John Wiley \& Sons, Ltd.

Received 26 July 1997

Revised 24 March 1998 
the Cosserat and Toupin-Mindlin theories were developed for linear elastic materials. Afterwards, non-local theories for plastic materials have been developed by, among others, Aifantis, ${ }^{4}$ Fleck and Hutchinson. ${ }^{5,6}$ Fleck-Hutchinson strain gradient plasticity theory ${ }^{6}$ falls within the Toupin-Mindlin framework. Interest in non-local continuum plasticity theories has been rising recently, due to an increasing number of observed size effects in plasticity phenomena. The reader is referred to a recent review article $^{6}$ for a summary of the experimental evidence.

The purpose of the current paper is to report the details of a finite element implementation of the Toupin-Mindlin formulation of strain gradient theories. The Toupin-Mindlin formulation furnishes strain gradients and higher order stresses which enter the principle of virtual work as work conjugates. The strain gradient quantities are the second-order spatial derivatives of displacement; therefore, in order to guarantee convergence of a displacement-based finite element analysis upon mesh refinement, the interpolation of displacement should exhibit $C^{1}$-continuity, i.e. both displacement and its first-order derivatives are required to be continuous across inter-element boundaries. ${ }^{7}$ For example, the use of Specht's triangular element ${ }^{8}$ for the special case of couple stress theory was examined. ${ }^{9}$ The element contains displacement derivatives as extra nodal degrees of freedom (denoted as DOF subsequently), and $C^{1}$-continuity is satisfied only in a weak averaged sense along each side of the element; therefore, the element is not a strict $C^{1}$ element. Furthermore, the element fails to deliver an accurate pressure distribution for an incompressible, non-linear solid. ${ }^{9}$ There is a reliable rectangular $C^{1}$ element, ${ }^{7}$ but its shape is obviously a strong limitation.

The lack of robust $C^{1}$-continuous elements drove the development of various $C^{0}$-continuous elements for couple stress theory. ${ }^{9-11}$ The elements developed by Xia and Hutchinson ${ }^{9}$ and by Shu and Fleck ${ }^{10}$ have a rotation angle $\boldsymbol{\theta}$ as an extra DOF at each node. The elements developed by Herrmann ${ }^{11}$ are based on a mixed formulation which takes the couple stresses as extra nodal DOF; it is not obvious how Herrmann's elements ${ }^{11}$ can be adapted to non-linear problems.

For the more general Toupin-Mindlin strain gradient theory, the straightforward generalization of the elements developed by Xia and Hutchinson ${ }^{9}$ and by Shu and Fleck ${ }^{10}$ would require roughly 3 times as many nodal DOF as an element for a conventional theory. There is a strong motivation to devise suitable elements with a smaller increase of computational cost. Six different types of two-dimensional finite elements are developed in this paper. They all use $C^{0}$-continuous shape functions, and they all pass a patch test. Computations are performed for two example problems and the performance of the new elements is evaluated by comparing numerical results with analytical solutions. Several types of element are capable of producing accurate results, with only a moderate increase in computational cost compared with elements for conventional theories. The best-performing element has been used to analyse the shear deformation of a bicrystal obeying an elastic-viscoplastic strain gradient crystal plasticity formulation and is found to generate satisfactory results. ${ }^{12}$

In this paper, deformations are assumed to be infinitesimally small. A subscript index, unless stated otherwise, takes a value of $1-3$ and repeated indices implies a summation over 1-3. A Cartesian reference-frame is employed throughout the paper, and a bold character represents a vector or tensor.

\section{REVIEW OF LINEAR ELASTIC STRAIN GRADIENT THEORY}

Toupin $^{2}$ and Mindlin ${ }^{3}$ developed a theory of linear elasticity whereby the strain energy density per unit volume depends upon both strain $\varepsilon_{i j} \equiv\left(u_{i, j}+u_{j, i}\right) / 2$ and strain gradient $\eta_{i j k} \equiv u_{k, i j}$. Here 
$\mathbf{u}$ is the displacement field and a comma represents partial differentiation with respect to a Cartesian co-ordinate. Note that $\varepsilon_{i j}=\varepsilon_{j i}$ and $\eta_{i j k}=\eta_{j i k}$. In addition to Cauchy stress $\sigma_{i j}$, this theory furnishes higher-order stress $\tau_{i j k}\left(=\tau_{j i k}\right)$ which is work conjugate to the strain gradient $\eta_{i j k}$. Fleck and Hutchinson ${ }^{6}$ extended the Toupin-Mindlin theory by including plasticity.

The principle of virtual work can be expressed as ${ }^{6}$

$$
\int_{v}\left[\sigma_{i j} \delta \varepsilon_{i j}+\tau_{i j k} \delta \eta_{i j k}\right] \mathrm{d} V=\int_{v}\left[b_{k} \delta u_{k}\right] \mathrm{d} V+\int_{s}\left[f_{k} \delta u_{k}+r_{k} D \delta u_{k}\right] \mathrm{d} S
$$

for an arbitrary displacement increment $\delta \mathbf{u}$. Here $b_{k}$ is the body force per unit volume of the body $V$ while $f_{k}$ and $r_{k}$ are the truncation and double stress traction per unit area of the surface $S$, respectively. They are in equilibrium with the Cauchy stress $\sigma_{i j}$ and the higher-order stress $\tau_{i j k}$ according to

$$
\begin{gathered}
b_{k}+\left(\sigma_{i k}-\tau_{j i k, j}\right)_{, i}=0 \\
f_{k}=n_{i}\left(\sigma_{i k}-\tau_{j i k, j}\right)+n_{i} n_{j} \tau_{i j k}\left(D_{p} n_{p}\right)-D_{j}\left(n_{i} \tau_{i j k}\right)
\end{gathered}
$$

and

$$
r_{k}=n_{i} n_{j} \tau_{i j k}
$$

In the above equations, $D_{j}()=.\left(\delta_{j k}-n_{j} n_{k}\right) \partial(.) / \partial x_{k}$ is a surface-gradient operator and $D()=.n_{k} \partial(.) / \partial x_{k}$ is surface normal-gradient operator. $n_{i}$ is the $i$ th component of the unit surface normal vector.

The strain gradient theory dictates that, in order to have a unique solution, six boundary conditions must be independently prescribed at any point on the surface of the body, i.e. $f_{k}$ or $u_{k}$, and $r_{k}$ or $D u_{k}$. The extra boundary conditions on $r_{k}$ or $D u_{k}$ are characteristic of the strain gradient theory and, as can be seen for a specific example in the Appendix, they imply strain continuity across the interface between two dissimilar materials.

The constitutive law governing the stress $\sigma_{i j}$ and the higher-order stress $\tau_{i j k}$ for an elastic solid is derived through $\sigma_{i j}=\partial w / \partial \varepsilon_{i j}$ and $\tau_{i j k}=\partial w / \partial \eta_{i j k}$ where $w$ is the strain energy density per unit volume. Mindlin $^{3}$ showed that for a general isotropic linear hyper-elastic solid, $w$ can be expressed as

$$
w=\frac{1}{2} \lambda \varepsilon_{i i} \varepsilon_{j j}+\mu \varepsilon_{i j} \varepsilon_{i j}+a_{1} \eta_{i j j} \eta_{i k k}+a_{2} \eta_{i i k} \eta_{k j j}+a_{3} \eta_{i i k} \eta_{j j k}+a_{4} \eta_{i j k} \eta_{i j k}+a_{5} \eta_{i j k} \eta_{j k i}
$$

in terms of the invariants of the second-order strain tensor and the third-order strain gradient tensor. Here $\lambda$ and $\mu$ are the standard Lame constants and the five $a_{n}$ are additional constants of dimension stress times length squared. $w$ can also be rewritten in the following canonical form: 6

$$
w=\frac{1}{2} \lambda \varepsilon_{i i} \varepsilon_{j j}+\mu \varepsilon_{i j} \varepsilon_{i j}+\mu\left(c_{1} \eta_{i j k}^{\prime(1)} \eta_{i j k}^{\prime(1)}+c_{2} \eta_{i j k}^{(2)} \eta_{i j k}^{\prime(2)}+c_{3} \eta_{i j k}^{\prime(3)} \eta_{i j k}^{\prime(3)}+c_{4} \eta_{i j k}^{\mathrm{H}} \eta_{i j k}^{\mathrm{H}}+c_{5} \eta_{i j k}^{\mathrm{H}} \eta_{j k i}^{\prime(3)}\right)
$$

where $\boldsymbol{\eta}^{\prime(i)}$ and $\boldsymbol{\eta}^{\mathrm{H}}$ are the bases of a unique orthogonal decomposition of $\boldsymbol{\eta}$ introduced by Smyshlyaev and Fleck. ${ }^{13} \boldsymbol{\eta}^{\mathrm{H}}$ is the hydrostatic part of $\boldsymbol{\eta}$ while $\boldsymbol{\eta}^{\prime(i)}$ are the three orthogonal bases of the deviatoric part $\boldsymbol{\eta}^{\prime}=\boldsymbol{\eta}-\boldsymbol{\eta}^{\mathrm{H}}$. For an incompressible solid, $\boldsymbol{\eta}^{\mathrm{H}} \equiv \mathbf{0}$. The details of the decomposition are omitted here for the sake of brevity. The constants $c_{n}$ have the dimensions of length squared. In the following context, $c_{n}$ will be referred to as constitutive 'length scales'. 


\section{FINITE ELEMENT IMPLEMENTATION}

\section{Weak form of the principle of virtual work}

Second-order derivatives of displacement appear in the principle of virtual work (equation (1)), implying that displacement-based elements of $C^{1}$-continuity are generally necessary in a finite element procedure. ${ }^{7}$ However, no robust $C^{1}$-continuous elements are available in the literature for application to the strain gradient theory outlined above. ${ }^{9}$ In this paper, $C^{0}$-continuous elements of mixed type are devised. We will first derive a weak form of the principle of virtual work suitable for finite element implementation using $C^{0}$-shape functions. The strategy is to introduce additional nodal degrees of freedom and to enforce the kinematic constraints between displacement and strain by Lagrange multipliers.

We begin by developing a modified virtual work statement involving only first-order gradients of kinematic quantities. Consider a strain gradient solid with a stress field satisfying the equilibrium relations (2)-(4). We introduce a second-order tensor $\psi$ and a related third-order tensor $\hat{\boldsymbol{\eta}}$ such that $\hat{\eta}_{i j k}$ is defined by $\hat{\eta}_{i j k} \equiv\left(\psi_{j k, i}+\psi_{i k, j}\right) / 2$. The equilibrium equations (2)-(4) may be re-written in weak form, by making use of Stokes' surface divergence theorem, as

$$
\begin{aligned}
\int_{v}[\boldsymbol{\sigma}: \delta \boldsymbol{\varepsilon}+\tau: \delta \hat{\boldsymbol{\eta}}+\vec{\nabla} \tau:(\delta \boldsymbol{\psi}-\vec{\nabla} \delta \mathbf{u})] \mathrm{d} V= & \int_{v}[\mathbf{b} \cdot \delta \mathbf{u}] \mathrm{d} V+\int_{s}[\mathbf{f} \cdot \delta \mathbf{u}+\mathbf{n r}: \delta \boldsymbol{\psi}] \mathrm{d} S \\
& +\int_{s}(\mathbf{n} \cdot \tau-\mathbf{n r}):(\delta \boldsymbol{\psi}-\vec{\nabla} \delta \mathbf{u}) \mathrm{d} S
\end{aligned}
$$

for arbitrary variations of $\delta \mathbf{u}$ and $\delta \psi$. Here $\vec{\nabla}$ is the standard forward gradient operator. If $\psi$ is subjected strictly to the constraint of

$$
\delta \psi=\vec{\nabla} \delta \mathbf{u}
$$

everywhere, then (7) degenerates to (1) and $\hat{\boldsymbol{\eta}}$ becomes identically the strain gradient $\boldsymbol{\eta}$ as defined in the previous section. However, as previously discussed, the strict enforcement of this constraint will demand $C^{1}$-continuous elements. Therefore, to facilitate the use of convenient $C^{0}$-continuous elements, the constraint (8) is enforced in the following weighted residual manner:

$$
\int_{v}\left(\psi_{j k}-u_{k, j}\right) \delta \tau_{i j k, i} \mathrm{~d} V=0 \quad(\text { no sum on } j \text { and } k \text { ) }
$$

for an arbitrary variation of the Lagrange multipliers $\delta \tau_{i j k, i}$. Satisfaction of (8) in the volume average sense by application of (9) ensures that the third term on the right-hand side of (7) is negligible. Finally, by denoting the Lagrange multipliers $\vec{\nabla} \cdot \tau$ as $\rho$ the modified virtual work statement (7) becomes

$$
\int_{v}[\boldsymbol{\sigma}: \delta \boldsymbol{\varepsilon}+\tau: \delta \hat{\boldsymbol{\eta}}+\boldsymbol{\rho}:(\delta \boldsymbol{\psi}-\overrightarrow{\boldsymbol{\nabla}} \delta \mathbf{u})] \mathrm{d} V \approx \int_{v}[\mathbf{b} \cdot \delta \mathbf{u}] \mathrm{d} V+\int_{s}[\mathbf{f} \cdot \delta \mathbf{u}+\tilde{\boldsymbol{\gamma}}: \delta \boldsymbol{\psi}] \mathrm{d} S
$$

where the second-order tensor $\tilde{\mathbf{r}}$ denotes $\mathbf{n r}$ for later convenience and (9) becomes

$$
\int_{v}\left(\psi_{j k}-u_{k, j}\right) \delta \rho_{j k} \mathrm{~d} V=0 \quad(\text { no sum on } j \text { and } k \text { ) }
$$


Henceforth, we shall refer to $\psi$ as the relaxed displacement gradient in contrast to the true displacement gradient $\vec{\nabla} \mathbf{u}$ and similarly, $\hat{\boldsymbol{\varepsilon}}=\left(\psi+\psi^{\mathrm{T}}\right) / 2$ as the relaxed strain in contrast to the true strain $\varepsilon$. Then, the Lagrange multiplier technique (9) ensures that $\psi$ is a good approximation to $\vec{\nabla} \mathbf{u}$. Consequently, the relaxed strain $\hat{\boldsymbol{\varepsilon}}$ is a good approximation to $\boldsymbol{\varepsilon}$, and the relaxed strain gradient $\hat{\boldsymbol{\eta}}$ is a good approximation to the true strain gradient $\boldsymbol{\eta}$. Both $\boldsymbol{\psi}$ and $\mathbf{u}$ are treated as independent nodal degrees of freedom. Only first-order derivatives of the DOF are involved in the weak form of the principle of virtual work equation (10), therefore $C^{0}$-continuous interpolation of both $\mathbf{u}$ and $\psi$ are sufficient to ensure convergence of the finite element procedure. The virtual work statement (10) is suitable for a compressible solid. In the incompressible limit, the mean stress $\sigma_{m}=\sigma_{k k} / 3$ is excluded from $\boldsymbol{\sigma}$ and treated as an additional Lagrange multiplier to enforce $\varepsilon_{k k}=0$, in a well-documented way for solids without strain gradient effects. ${ }^{7}$

As stated in the previous section, to obtain a unique solution for a boundary value problem, six independent boundary conditions should be prescribed on the surface for a three-dimensional problem (and four independent boundary conditions for a two-dimensional problem). While the boundary conditions associated with nodal DOF of displacement are easily set, the way to prescribe boundary conditions associated with nodal DOF $\psi$ needs to be specially addressed. Let us designate a prescribed quantity on the surface with a superscript*. Then, for the case of pure traction-loading, stress-tractions $\mathbf{f}^{*}$ and double stress tractions $\mathbf{r}^{*}$ are applied, giving

$$
\mathbf{f}=\mathbf{f}^{*} \quad \text { and } \quad \tilde{\mathbf{r}}=\mathbf{n r} *
$$

For displacement-loading, displacement $\mathbf{u}^{*}$ and displacement gradient Du* are prescribed as follows. Denote $\mathbf{t}$ and $\mathbf{s}$ as two orthogonal surface tangential unit vectors which form a triad with the normal unit vector $\mathbf{n}$. The boundary conditions are specified as

$$
\mathbf{u}=\mathbf{u}^{*} \text { and } \boldsymbol{\psi}=\mathbf{n D u} \mathbf{u}^{*}+\mathbf{t D}_{t} \mathbf{u}^{*}+\mathbf{s D}_{s} \mathbf{u}^{*}
$$

where $\mathrm{D}_{t}()=.\mathbf{t} \cdot \vec{\nabla}($.$) and \mathrm{D}_{s}()=.\mathbf{s} \cdot \vec{\nabla}($.$) are the surface tangential gradient operators. \mathrm{D}_{t} \mathbf{u}^{*}$ are $\mathrm{D}_{s} \mathbf{u}^{*}$ are known if the surface displacement $\mathbf{u}^{*}$ is prescribed. With this boundary condition, there will be no error introduced in (10) when the third term on the right-hand side of (7) is dropped.

Now, consider mixed boundary conditions. For the case of $\mathbf{f} *$ and Du* given, we specify $\mathbf{f}=\mathbf{f}^{*}$ and

$$
\mathbf{n} \cdot \boldsymbol{\psi}=\mathrm{Du}^{*}, \quad \mathbf{s} \cdot \tilde{\mathbf{r}}=\mathbf{0} \text { and } \mathbf{t} \cdot \tilde{\mathbf{r}}=\mathbf{0}
$$

Alternatively, if $\mathbf{u}^{*}$ and $\mathbf{r}^{*}$ are prescribed, then we enforce $\mathbf{u}=\mathbf{u}^{*}$ and

$$
\mathbf{n} \cdot \tilde{\mathbf{r}}=\mathbf{r}^{*}, \quad \mathbf{s} \cdot \boldsymbol{\psi}=\mathrm{D}_{s} \mathbf{u}^{*} \quad \text { and } \mathbf{t} \cdot \boldsymbol{\psi}=\mathrm{D}_{t} \mathbf{u}^{*}
$$

Stipulation that $\mathbf{s} \cdot \boldsymbol{\psi}=\mathrm{D}_{s} \mathbf{u}^{*}$ and $\mathbf{t} \cdot \boldsymbol{\psi}=\mathrm{D}_{t} \mathbf{u}^{*}$ reduces the error in the approximation equation (10), associated with dropping the third term on the right-hand side of (7).

An iterative solution procedure ${ }^{14}$ is adopted to solve the system of linear equations for nodal DOF involving zero diagonal terms.

\section{Finite elements}

In this section we introduce six types of isoparametric element for the strain gradient solid; they are sketched in Figure 1. We restrict attention to two-dimensional deformation in the $\left(x_{1}, x_{2}\right)$ plane, and assume that subscript indices range from 1 to 2 . 

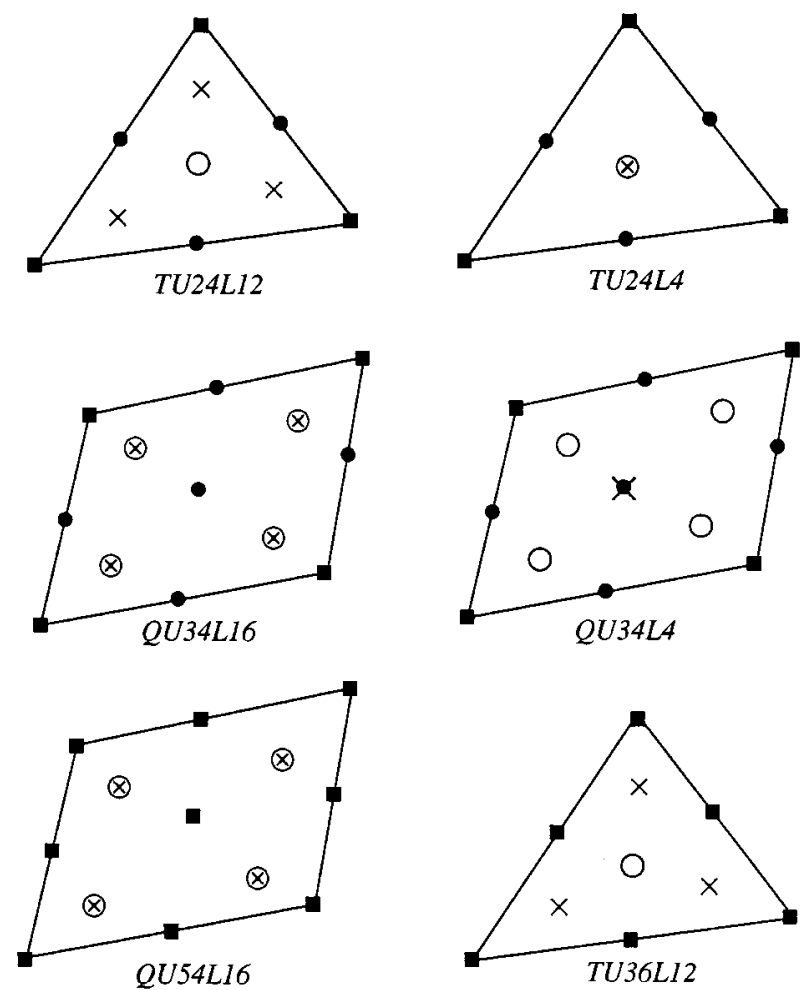

DOF at $\bullet: \mathrm{u}_{1}, \mathrm{u}_{2}, \Psi_{11}, \psi_{21}, \psi_{12}, \psi_{22}$
DOF at $\bullet: \mathrm{u}_{1}, \mathrm{u}_{2}$

Lagrange multipliers at $x: \rho_{11}, \rho_{21}, \rho_{12}, \rho_{22}$

Lagrange multiplier (for incompressible solid) at $O: \sigma_{\mathrm{m}}$

Figure 1. Sketch of six types of element

First, we explain the code used to label each type of element, for example TU24L12. Triangular elements are designated by a leading letter $T$, and quadrilateral elements by a leading letter $Q$. The nodal degrees of freedom are of displacement-type (u or $\psi)$, and the total number of degrees of freedom $n$ per element is written as $U n$. Finally, the number $m$ of Lagrange multipliers $(\boldsymbol{\rho})$ is designated Lm. Hence, element TU24L12 is a triangular element, with 24 nodal degrees of freedom at 12 Lagrange multipliers $\rho_{i j}$. The six types of element described below fall within three classes:

(i) triangular elements where the number of DOF is different for corner nodes and for mid-side nodes;

(ii) quadrilateral elements where the number of DOF is different for corner nodes and for mid-side nodes;

(iii) triangular and quadrilateral elements which possess the same number of DOF for all nodes. 
(i) TU24L12 and TU24L4 elements

Both elements TU24L12 and TU24L4 are six-noded isoparametric triangular elements. The location of each node in terms of the area co-ordinates for a triangle is standard. It has six degrees of freedom, $u_{1}, u_{2}, \psi_{11}, \psi_{21}, \psi_{12}$ and $\psi_{22}$ at each corner node and two degrees of freedom, $u_{1}$ and $u_{2}$ at each 'mid-side' node. The total number of degrees of freedom per element is 24 . Displacements $u_{i}$ are interpolated using standard quadratic shape functions in terms of the area co-ordinates. Relaxed displacement gradients $\psi_{i j}$ are interpolated linearly. The different interpolation is motivated by the consideration that the true displacement gradients will depend linearly on the co-ordinates if all sides of the element are straight.

In a TU24L12 element, four nodal values of Lagrange multiplier $\left(\rho_{11}, \rho_{21}, \rho_{12}\right.$ and $\left.\rho_{22}\right)$ exist at each quadrature point of the three-point Laursen and Gellert ${ }^{15}$ integration rule. The Lagrange multipliers are interpolated linearly between these three points. Since this triangular element has 12 Lagrange multipliers $\rho_{i j}$ and 24 DOF it is designated the code TU24L12. An alternative element (TU24L4) has also been constructed with 4 constant Lagrange multipliers within the element.

For both types of elements, the stiffness matrix is calculated as follows. The deviatoric Cauchy stress terms and the higher-order stress terms in the principle of virtual work are integrated by the three-point integration scheme. ${ }^{15}$ For a compressible solid, the pressure term is also integrated by this scheme. For an incompressible solid, the pressure is treated as an additional Lagrange multiplier, is assigned a constant value throughout the element ${ }^{7}$ and is integrated by one-point quadrature. The Lagrange multiplier $\rho_{i j}$ terms are integrated by full three-point integration.

It is known that for a finite element mesh, the total number of independent nodal DOF (denoted as $n_{\mathrm{d}}$ ) should exceed the total number (denoted as $n_{\lambda}$ ) of all kinematic constraints (including incompressibility) in order to generate numerically stable solutions. ${ }^{7}$ Although the element TU24L12 performs satisfactorily in some of the numerical tests conducted, it often violates the numerical stability condition for a mesh with a large number of elements. On the other hand, element TU24L4 generally satisfies the stability condition. Numerical experiments have shown that TU24L4 used alone or in a proper combination with TU24L12 satisfies the stability condition and can generate reasonably accurate results.

\section{(ii) QU34L16 and QU34L4 elements}

These are nine-noded isoparametric quadrilateral elements, as shown in Figure 1. The location of each node in terms of the local co-ordinates $\left(\xi_{1}, \xi_{2}\right)\left(-1 \leqslant \xi_{i} \leqslant 1\right)$ is standard. There are six DOF, $u_{1}, u_{2}, \psi_{11}, \psi_{21}, \psi_{12}$ and $\psi_{22}$ at each corner node, and 2 DOF, $u_{1}$ and $u_{2}$ at each of the remaining five nodes. The total number of nodal DOF is 34 . The two displacements are interpolated by the conventional biquadratic Lagrangian shape functions and the relaxed displacement gradients $\psi_{i j}$ are interpolated bilinearly.

In a QU34L16 element, the four Lagrange multipliers $\rho_{i j}$ are interpolated bilinearly between nodal values given at the four quadrature points of a $2 \times 2$ Gauss integration scheme. There are a total of 16 nodal values of Lagrange multipliers $\rho_{i j}$ and therefore the element is denoted by QU34L16. A slightly modified element (QU34L4) uses four Lagrange multipliers $\rho_{i j}$, assumed constant throughout the element.

The deviatoric stress terms, the pressure terms for a compressible solid and the higher-order stress terms are all integrated using full integration of $3 \times 3$ Gauss quadrature. For the case of an incompressible solid, pressure forms an additional Lagrange multiplier, and is interpolated 
bilinearly between four nodal values at the Gauss points of a $2 \times 2$ quadrature scheme. ${ }^{7} 2 \times 2$ Gauss quadrature is used to integrate all terms involving any Lagrange multiplier.

Meshes consisting solely of QU34L16 elements often violate the numerical stability condition, for both compressible and incompressible solids. On the other hand, the QU34L4 elements satisfy the stability condition and have been found to work well for both compressible and incompressible solids. A combination of QU34L16 and QU34L4 also perform satisfactorily.

(iii) QU54L16 and TU36L12 elements

Element QU54L16 is identical to QU34L16 except that now there are six DOF, $u_{1}, u_{2}, \psi_{11}, \psi_{21}, \psi_{12}$ and $\psi_{22}$ at every node and all DOF are interpolated using standard biquadratic Lagrange shape functions. The element is denoted as QU54L16 because the total number of DOF per element is 54. Regardless of the degree of compressibility, meshes of this element satisfy the numerical stability condition. The same integration scheme is used as that described for the element QU34L16. Though the element performs well in the numerical tests conducted, it suffers from the drawback that, for a mesh with a significant number of such elements, there are roughly twice as many nodal DOF as exist in a mesh with the same number of QU34L4 elements.

A six-noded triangular element TU36L12 was also developed. It employs quadratic interpolation for $\mathbf{u}, \boldsymbol{\psi}$ and linear interpolation for $\boldsymbol{\rho}$. A mesh consisting solely of these elements marginally satisfies the stability condition when the material is compressible but fails to satisfy the stability condition for an incompressible solid. This element performed poorly during a $2 \mathrm{D}$ test calculation (see section below), and although it is able to give adequate results in a $1 \mathrm{D}$ simulation, it suffers from the same drawback as that of QU54L16 as discussed in the previous paragraph. Hence the use of this element alone is not recommended.

\section{NUMERICAL TESTS}

Three types of numerical test were performed: (1) patch tests for a 2-D mesh of elements under uniform loading; (2) 1-D boundary layer analysis and (3) 2-D stress analysis for a plate containing a hole. In all cases, plane-strain deformations were assumed and body forces were absent. In the presentation of results, nodal values of stress and strain were extrapolated from Gauss point values by a smoothing scheme described in Appendix I.

\section{Patch test}

All types of element were subjected to patch tests as shown in Figure 2. In each case the mesh was loaded in uniaxial tension by end stress-tractions, as indicated in the figure. The material was discretized into a mesh of $n \times n$ identical quadrilateral elements or $2 \times n \times n$ identical triangular elements, with $n$ ranging from 1 to 5 . Triangular elements were obtained by sub-dividing each square along a diagonal (dashed line).

The elements TU24L12 and QU34L16 passed all patch tests for any choice of constitutive parameters, including the case of all length scales $c_{i}$ being identically zero.

The remaining elements QU34L4, TU24L4, QU54L16 and TU36L12 give the correct stress and strain states when at least one of the material length scales $c_{i}$ is finite. For the limiting case of all $c_{i}=0$ (i.e. the material has degenerated to a conventional solid), these four elements have 

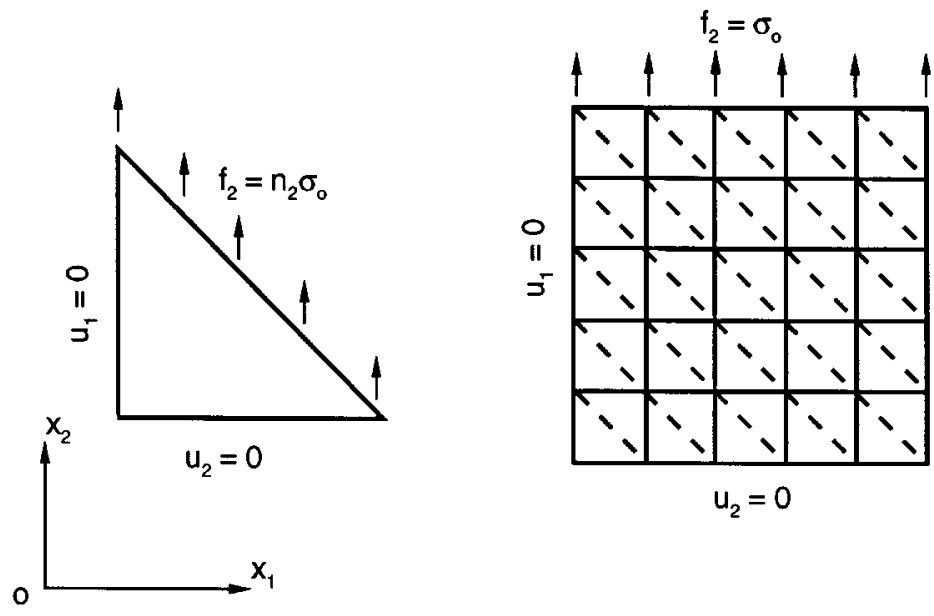

Figure 2. Mesh and the boundary conditions for the patch test

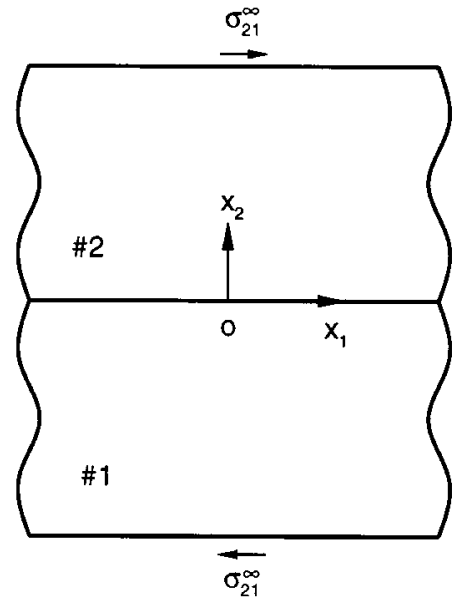

(a)

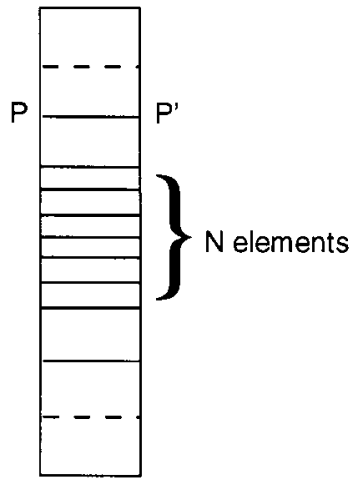

(b)

Figure 3. (a) Notation and geometry of a bimaterial under uniform shear; (b) sketch of the mesh

zero-energy modes. This is because the number of nodal relaxed displacement gradients $\psi_{i j}$ exceeds that of the linearly independent kinematic constraints and the vanishing length scales render the derivatives of $\psi_{i j}$ indeterminate. For the particular choice $c_{1}=0$, with at least one of the remaining $c_{i}$ taking on a finite value, the solution for the relaxed displacement gradient $\psi_{i j}$ depends upon the value chosen for the accelerator parameter of iterative solution procedure laid out in Reference 13.

We conclude that all six types of element pass the patch tests, if at least one $c_{i} \neq 0$. 
Boundary layer analysis

Higher-order gradient theories predict the existence of boundary layers adjacent to inhomogeneities such as interfaces. Consider, for example, a bimaterial composed of two perfectly bonded half planes of elastic strain gradient solids, subjected to a remote shear stress $\sigma_{12}^{\infty}$ as shown in Figure 3(a). An analytical solution is presented in the Appendix II: for the strain gradient solid specified by equations (1)-(6) the shear strain $\varepsilon_{12}$ has a continuous but nonuniform distribution within a boundary layer adjacent to the interface. A similar solution was obtained for a couple stress theory solid. ${ }^{5}$ In a specific quantitative example, we shall make the following arbitrary choice of constitutive parameters. The shear modulus $\mu_{1}$ of material 1 is taken
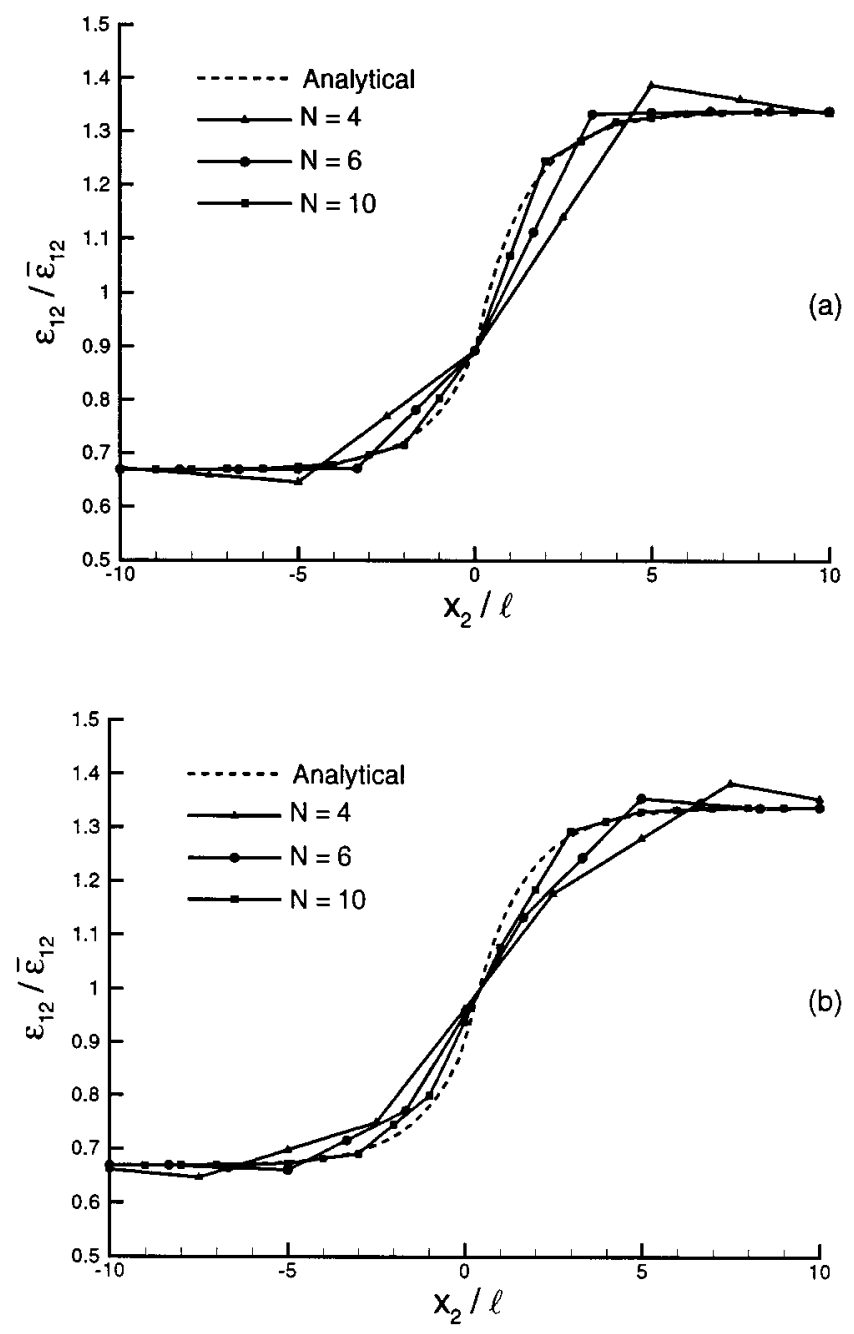

Figure 4. Accuracy study of various elements in the boundary layer analysis: (a) strain $\varepsilon_{12}$ by QU34L16; (b) strain $\varepsilon_{12}$ by QU34L4; (c) relaxed strain $\hat{\varepsilon}_{12}$ by QU34L4 


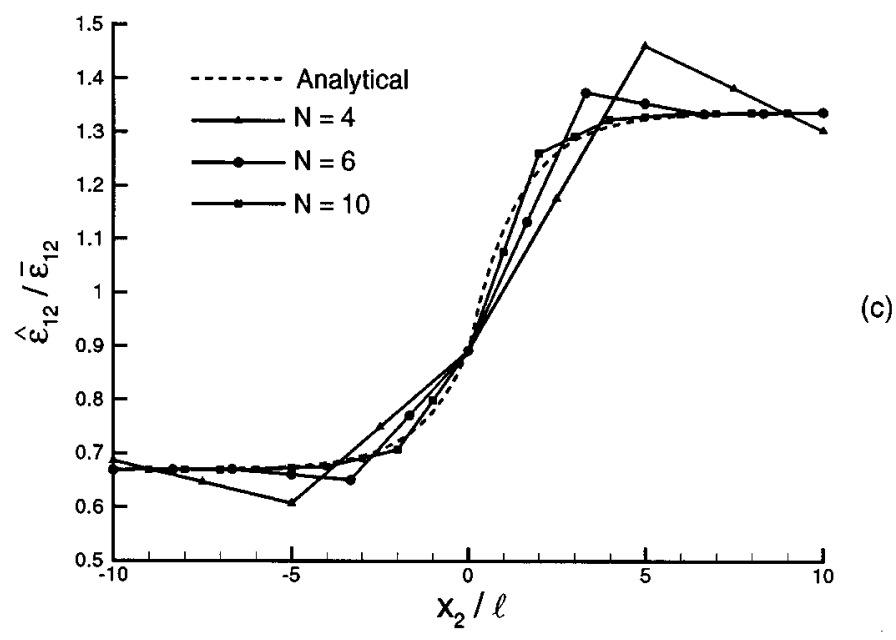

Figure 4. (Continued)

to be twice that of material 2. For both materials, the constants $c_{1}$ to $c_{4}$ (as defined in equation (6)) are equal to $\ell^{2}$ while $c_{5}=0$. We shall examine the ability of the six types of element described above to predict this boundary layer.

The finite element mesh, sketched in Figure 3(b), is fixed at a width of $\ell$ in the $x_{1}$-direction, and a length of $50 \ell$ in the $x_{2}$-direction on each side of the interface. A central region of length $10 \ell$ on each side of the interface is discretized into $N / 2$ identical quadrilateral elements. The two remaining end regions of the mesh are each divided into 10 quadrilateral elements. (Triangular elements are obtained by sub-dividing the quadrilaterals along a diagonal.) The following periodic boundary conditions are applied along the left- and right-hand sides of the mesh to enforce uniformity of deformation in the $x_{1}$-direction. For any pair of nodes $P$ and $P^{\prime}$ which are equally distanced from the interface and do not lie on the top or bottom surface, each nodal DOF at $P$ is equal to its counterpart at $P^{\prime}$ while the corresponding work-conjugate nodal force or double force at $P$ equals that at $P^{\prime}$ in magnitude but has an opposite sign. For any surface node, the double forces vanish while the nodal forces are constructed from the prescribed surface traction. For this one-dimensional problem, all types of element gave converged solutions. We evaluate below the relative performance of the elements by examining the accuracy of solution as a function of $N$.

The shear strain obtained using QU34L16 elements is plotted in Figure 4(a) after normalization by the average shear strain of $\bar{\varepsilon}_{12}=\sigma_{12}^{\infty}\left(\mu_{1}+\mu_{2}\right) /\left(2 \mu_{1} \mu_{2}\right)$. The plot of the relaxed shear strain, $\hat{\varepsilon}_{12}=\left(\psi_{12}+\psi_{21}\right) / 2$, is indistinguishable from that of the true shear strain and is not explicitly shown. It is clear from the figure that the finite element solution converges quickly to the exact solution with increasing refinement of mesh, $N$. It is interesting to note that the strain calculated at the interface is accurate even for a very coarse mesh $(N=4)$. The element TU24L12 gives comparable performance to QU34L16 for this 1-D problem.

Next, consider the element QU34L4. The shear strain and the relaxed shear strain are plotted in Figure 4(b) and 4(c), respectively. It can be seen that $\varepsilon_{12}$ and $\hat{\varepsilon}_{12}$ converge to the exact solution at a rate marginally slower than that demonstrated by element QU34L16. The accuracy is 


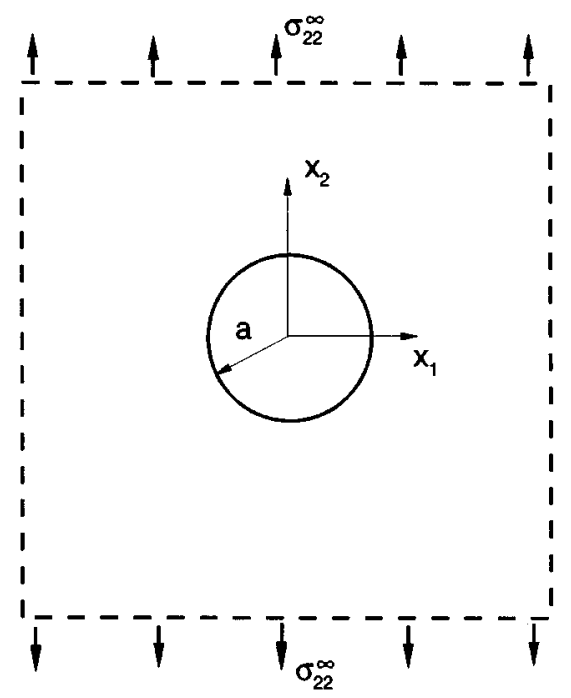

Figure 5. Notation and geometry of an infinite plane subjected to a remotely uniform tension

noticeably poorer only at the interface $\left(x_{2}=0\right)$. Similar performance tests were also carried out on elements TU24L4, TU36L12 and QU54L16; they showed similar convergence properties to those of QU34L4.

A combination of QU34L16 and QU34L4 elements was also used: the $N$ elements adjacent to the interface were chosen to be QU34L16 while the remaining were QU34L4 elements. The shear strain distribution is indistinguishable from that shown in Figure 4(a).

\section{Stress concentration due to a hole}

In order to examine the convergence properties of the various finite elements for a 2-D problem, we consider an infinite solid containing a circular cylindrical hole. The solid is subjected to remote uniform tension, as shown in Figure 5. An analytical solution was obtained ${ }^{16}$ for this problem for the special case of a couple stress solid, i.e. the strain energy density per unit volume $w$ depends upon strain and upon that part of strain gradients which can be expressed in terms of curvature $\chi_{i j}$ by

$$
w=\frac{1}{2} \lambda \varepsilon_{i i} \varepsilon_{j j}+\mu \varepsilon_{i j} \varepsilon_{i j}+2 \mu \ell^{2} \chi_{i j} \chi_{i j}
$$

Here, $\chi_{i j}$ is related to the rotation of material elements $\boldsymbol{\theta}$ by $\chi_{i j}=\theta_{i, j}=\frac{1}{2} e_{i p k} u_{k, p j}$. The problem is re-analysed using the elements developed here. We note that the couple stress constitutive law is recovered by setting the five constitutive length scales in equation (6) to be $c_{1}=0, c_{2}=3 \ell^{2} / 4$, $c_{3}=5 \ell^{2} / 8, c_{4}=\ell^{2} / 8$ and $c_{5}=-\ell^{2}$.

The symmetry of geometry and boundary conditions imply that a quarter mesh is adequate. All computations were carried out using the mesh shown in Figure 6 which has 720 quadrilateral elements and 2983 nodes. Triangular elements were generated by sub-dividing a quadrilateral element along a diagonal. The boundary conditions prescribed are illustrated in Figure 6. Results 

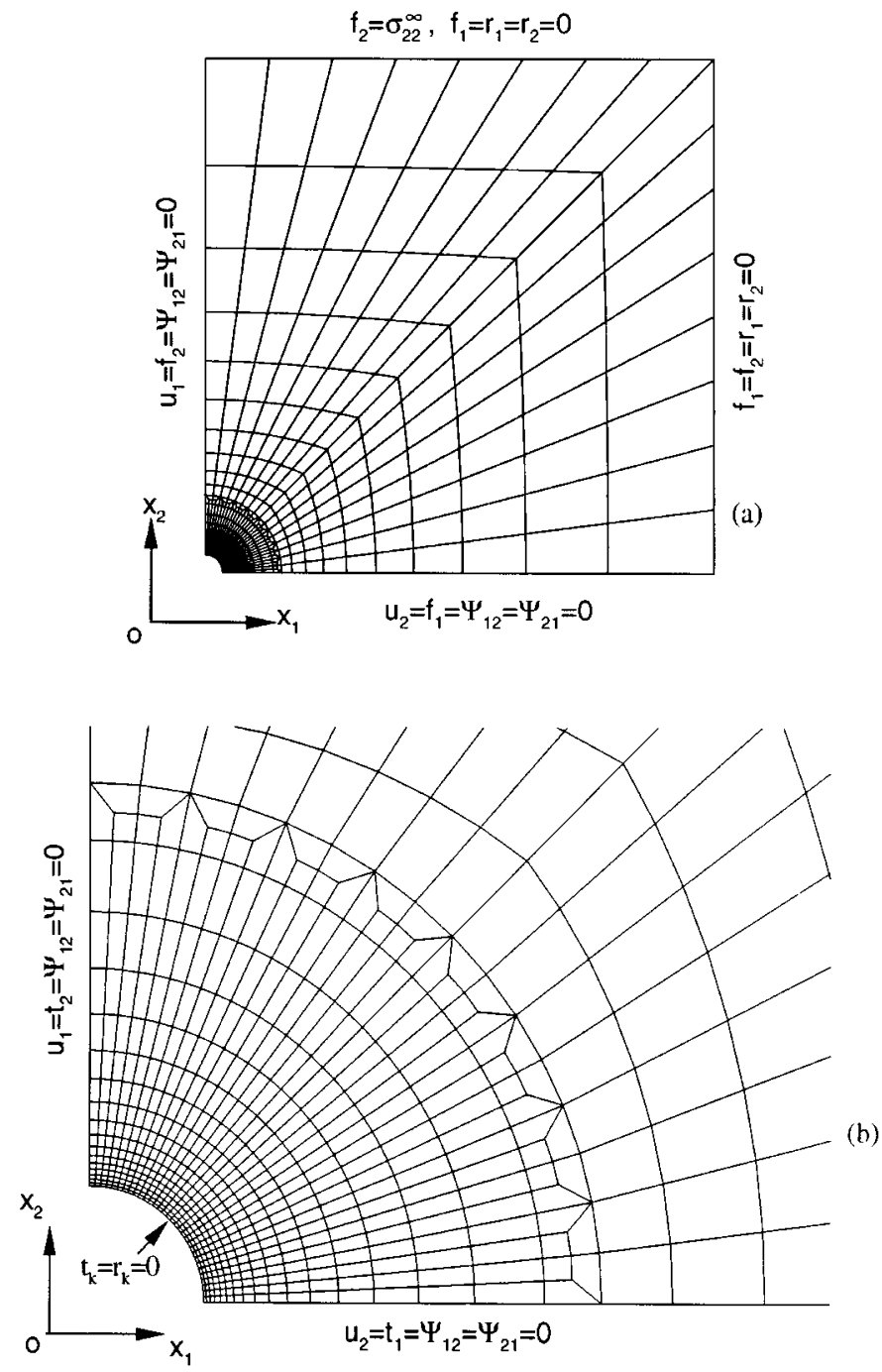

Figure 6. (a) Global view of a mesh used for the stress concentration problem as depicted in Figure 5. The mesh has 720 quadrilateral elements and 2983 nodes. Boundary conditions are indicated; (b) the close-up view of the fine mesh zone. Boundary conditions on the hole surface are included

are presented in the form of the stress concentration factor at the edge of the hole; values as a function of normalized hole radius $a / \ell$ are given in Table I for Poisson's ratio $v=0$ and in Table II for Poisson's ratio $v=0 \cdot 5$.

The elements QU34L4 and QU54L16 both satisfy the stability condition $\left(n_{\mathrm{d}} / n_{\lambda}>1\right)$ for both compressible and incompressible solids, and give accurate solutions to the problem. Of the two, a mesh of QU54L16 elements has roughly twice as many DOF as a mesh of QU34L4 elements, leading to a significantly higher computational cost for no significant increase in accuracy. In 
Table I. Stress concentration factor $(v=0)$

\begin{tabular}{|c|c|c|c|c|c|}
\hline$a / \ell$ & Analytical* & QU34L4 & QU54L16 & $\begin{array}{r}\text { QU34L4 + } \\
\text { QU34L16 }\end{array}$ & TU24L4 \\
\hline $10^{2}$ & $3 \cdot 000$ & $3 \cdot 006$ & $3 \cdot 010$ & $3 \cdot 009$ & $3 \cdot 003$ \\
\hline 10 & $2 \cdot 878$ & $2 \cdot 894$ & $2 \cdot 888$ & $2 \cdot 888$ & $2 \cdot 902$ \\
\hline 8 & $2 \cdot 824$ & $2 \cdot 843$ & $2 \cdot 834$ & $2 \cdot 834$ & $2 \cdot 849$ \\
\hline 6 & $2 \cdot 729$ & $2 \cdot 750$ & $2 \cdot 733$ & $2 \cdot 738$ & $2 \cdot 758$ \\
\hline 4 & $2 \cdot 545$ & $2 \cdot 568$ & $2 \cdot 549$ & $2 \cdot 552$ & $2 \cdot 577$ \\
\hline 3 & $2 \cdot 389$ & $2 \cdot 412$ & $2 \cdot 397$ & $2 \cdot 396$ & $2 \cdot 422$ \\
\hline 2 & $2 \cdot 169$ & $2 \cdot 188$ & $2 \cdot 176$ & $2 \cdot 176$ & $2 \cdot 201$ \\
\hline 1 & $1 \cdot 889$ & $1 \cdot 897$ & $1 \cdot 893$ & $1 \cdot 895$ & 1.912 \\
\hline
\end{tabular}

*Analytical solution obtained in Reference 16

Table II. Stress concentration factor $(v=0 \cdot 5)$

\begin{tabular}{|c|c|c|c|c|c|}
\hline$a / \ell$ & Analytical* & QU34L4 & QU54L16 & $\begin{array}{l}\text { QU34L4 + } \\
\text { QU34L16 }\end{array}$ & TU24L4 \\
\hline $10^{2}$ & $3 \cdot 000$ & $3 \cdot 006$ & $3 \cdot 005$ & $3 \cdot 024$ & $2 \cdot 975$ \\
\hline 10 & $2 \cdot 937$ & $2 \cdot 948$ & $2 \cdot 947$ & $2 \cdot 965$ & $2 \cdot 908$ \\
\hline 8 & $2 \cdot 908$ & $2 \cdot 920$ & $2 \cdot 918$ & 2.935 & $2 \cdot 876$ \\
\hline 6 & $2 \cdot 855$ & $2 \cdot 869$ & $2 \cdot 865$ & $2 \cdot 879$ & $2 \cdot 814$ \\
\hline 4 & $2 \cdot 743$ & $2 \cdot 760$ & $2 \cdot 752$ & $2 \cdot 761$ & $2 \cdot 685$ \\
\hline 3 & 2.639 & $2 \cdot 657$ & $2 \cdot 648$ & $2 \cdot 653$ & $2 \cdot 557$ \\
\hline 2 & $2 \cdot 476$ & $2 \cdot 492$ & $2 \cdot 483$ & $2 \cdot 486$ & $2 \cdot 351$ \\
\hline 1 & $2 \cdot 231$ & $2 \cdot 243$ & $2 \cdot 236$ & $2 \cdot 251$ & 1.927 \\
\hline
\end{tabular}

*Analytical solution obtained in Reference 16

addition, we found that for element QU34L4 (and similarly for TU24L4), contours of stress and strain based on nodal values calculated by linear local smoothing are distorted around a central part of the hole surface, but constant local smoothing (see Appendix I) damps out the zig-zag of the contours, as shown in Figure 7(a) and 7(b). The contours of strain $\varepsilon_{22}$ and the relaxed strain $\hat{\varepsilon}_{22}$ are mostly indistinguishable.

Element TU24L4 is adequate for a compressible solid, but for an incompressible solid it converges to an inaccurate distribution of pressure, particularly for $\ell=a$. The relatively poor performance of TU24L4 in this case could be due to the assumed uniformity of pressure within an element.

A mesh consisting only of elements QU34L16 or TU24L12 fails to converge for any $v$ and finite $a / \ell$, as the stability criterion $\left(n_{\mathrm{d}} / n_{\lambda}>1\right)$ is violated. The element TU36L12 is a borderline case: for the compressible solid $\left(n_{\mathrm{d}} / n_{\lambda} \approx 1 \cdot 02\right)$ the solution converges slowly; for the incompressible case $\left(n_{\mathrm{d}} / n_{\lambda} \approx 0.92\right)$ the solution does not converge.

We conclude that for a mesh made solely of one type of element, the optimal element is QU34L4. This conclusion is moderated by the fact that elements can be used in combination to give satisfactory results. For example, a combination of QU34L16 and QU34L4 was tried by 

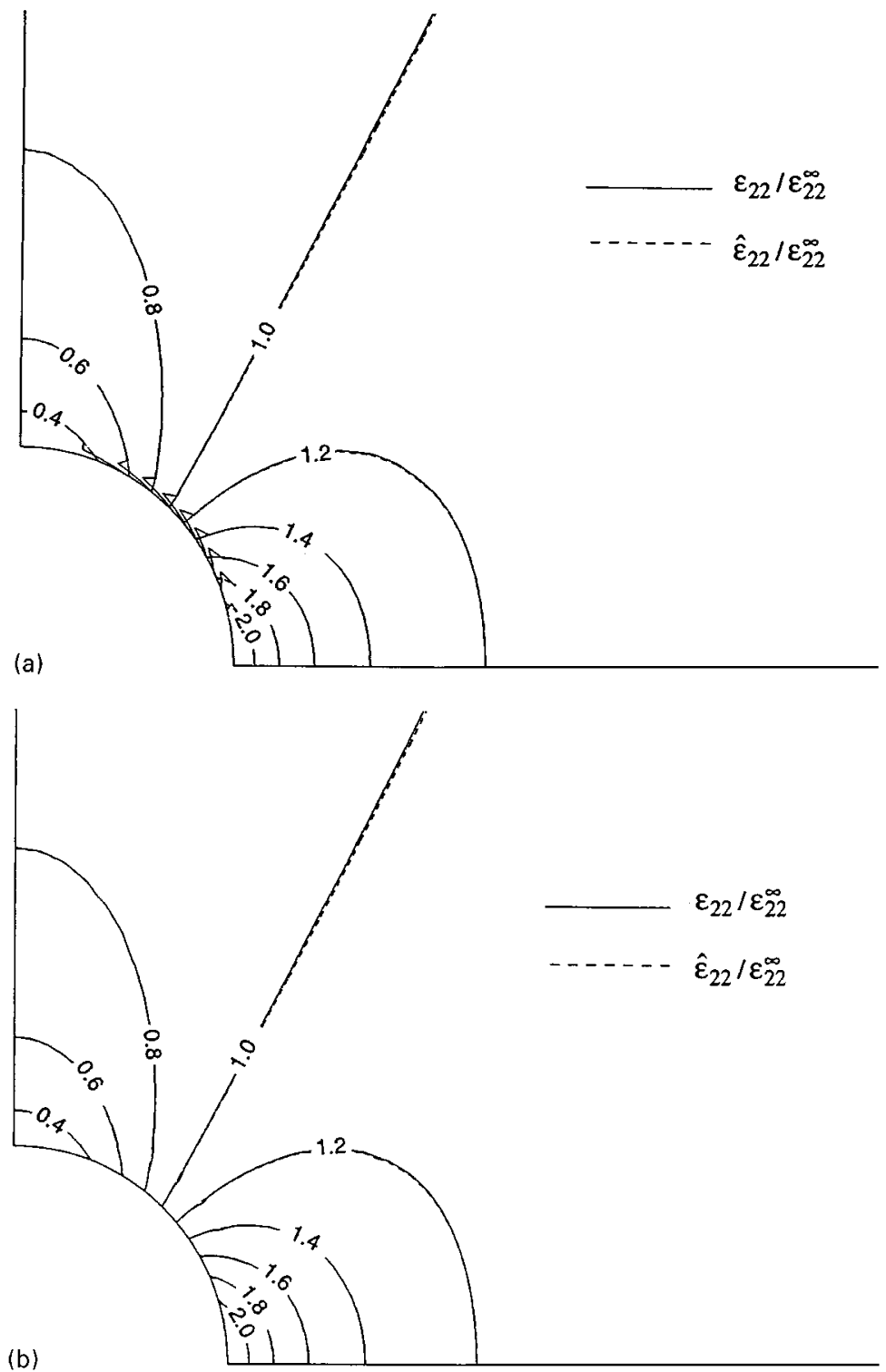

Figure 7. (a) Contours of normalized strain and relaxed strain around the hole with $\ell / a=1$. The material is incompressible. Nodal values of the strain are calculated with bilinear local smoothing. The mesh consists of QU34L4 elements; (b) Contours of normalized strain and relaxed strain around the hole with $\ell / a=1$. The material is incompressible. Nodal values of the strain are calculated with constant local smoothing and the surface values are extrapolated from inner nodes. The mesh consists of QU34L4 elements

taking the first four layers of elements of the mesh along the hole surface (see Figure $6(\mathrm{~b})$ ) as QU34L16 and the rest as QU34L4. This combination has $n_{\mathrm{d}} / n_{\lambda} \approx 1.24$ for $v=0.5$ and 2.05 for $v<0 \cdot 5$. Tables I and II indicate that the combination is able to produce the stress concentration factor with accuracy generally comparable to that by QU54L16. Echoing the finding in the 
boundary layer analysis in the previous section, we find for the hole problem that a small number of QU34L16 elements around a free surface gives slightly more accurate results at the surface. A word of caution: in practical applications, choosing the optimal 'small' number of QU34L16 elements is not an easy task and is problem-dependent.

Our numerical tests indicate that for a 2-D problem, if a mesh fails to satisfy the numerical stability condition $\left(n_{d} / n_{\lambda}>1\right)$, then the iterative equation solution procedure ${ }^{14}$ also fails to reach a converged solution. Furthermore, the closer $n_{\mathrm{d}} / n_{\lambda}$ is to unity, the more difficult it is to obtain a converged solution.

\section{CONCLUDING REMARKS}

A total of six types of triangular element and quadrilateral element have been developed for the Toupin-Mindlin framework of strain gradient theory. Displacement gradients are introduced as extra nodal degrees of freedom, and are termed the relaxed displacement gradients. The kinematic constraints between the relaxed displacement gradients and true gradients of displacement are enforced via Lagrange multipliers. For an incompressible solid, the pressure is treated as an additional Lagrange multiplier. This mixed formulation allows all the nodal degrees of freedom to be interpolated using standard $C^{0}$-continuous shape functions. All six types of element passed a patch test. Boundary value problems were solved using the various elements in order to evaluate their relative performance. Elements QU54L16 and QU34L4 yield the most accurate results with the latter one being computationally less expensive. TU24L4 is less accurate than QU34L4 for an incompressible solid. Element TU36L12 is not recommended for practical applications. Both QU34L16 and TU24L12 perform well in 1-D problems, but for general 2-D problems they can only be used in combination with QU34L4 and TU24L4. We conclude by recommending QU34L4 elements for practical applications.

\section{APPENDIX I}

\section{Smoothing scheme}

In this paper, nodal values of stress, strain and double stress were calculated from Gauss point values by element-wise (local) smoothing, followed by averaging over all elements adjoining a given node. Linear local smoothing ${ }^{17}$ was used in all elements except for QU34L4 and TU24L4. For these two elements, fluctuations in stress and strain occurred within elements adjacent to a free surface and were not damped-out by the linear smoothing. This can be attributed to the fact that the kinematic constraint equation (8) is enforced only at the geometrical center of an element and a free surface provides no additional constraints. For elements QU34L4 and TU24L4 smooth distributions of stress and strain were obtained by the following two-step scheme. First, constant local smoothing (taking the average over all Gauss points within an element and assigning the average onto all nodes of the element) was performed on the whole mesh, including surface elements. The values at any interior node were obtained by averaging the contributions from all elements sharing that node. Surface nodal values calculated by this step alone obviously would lose some accuracy, and so a second stage of smoothing was performed on these surface nodes. Linear extrapolation of values from two inner nodes was used to estimate surface nodal values, as sketched in Figure 8. For example, consider nodes at one end of a strip mesh sketched in Figure 8(a). A nodal value, denoted by $P_{1}$, at node 1 was calculated from the values at inner 


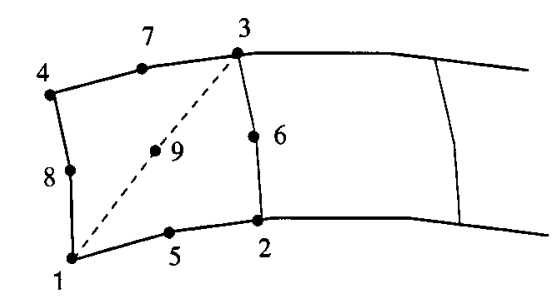

(a)

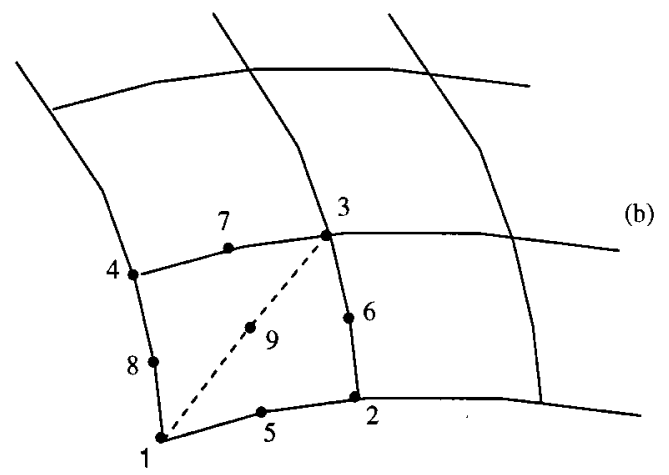

Figure 8. Illustrations for extrapolations from inner nodes onto surface nodes. The dashed lines represent a side of a triangular element: (a) nodes at the ends of a strip mesh used for the boundary layer analysis; (b) nodes around a corner of the mesh used for the hole problem.

nodes 3 and 9 by $P_{1}=2 * P_{9}-P_{3}$. Similarly, $P_{4}=2 * P_{9}-P_{2}$ and $P_{8}=2 * P_{9}-P_{6}$. For nodes around a corner of a mesh illustrated in Figure $8(\mathrm{~b})$, the surface nodal values were obtained by $P_{1}=2 * P_{9}-P_{3}, P_{8}=2 * P_{9}-P_{6}, P_{4}=2 * P_{7}-P_{3}, P_{5}=2 * P_{9}-P_{7}$ and finally $P_{2}=2 * P_{6}-P_{3}$. The scheme has been found to work well in the numerical tests.

\section{APPENDIX II}

\section{A bimaterial under remote shear}

An analytical solution is presented for a bimaterial, consisting of two perfectly bonded half planes of dissimilar linear elastic strain gradient solids. The bimaterial is subjected to a remote uniform shear stress $\sigma_{12}^{\infty}$, as shown in Figure 3(a). Here, we assume that material 1 laying below the interface has a shear modulus $\mu_{1}$, and an internal length scale $\ell_{1}$ by taking $c_{1}=c_{2}=c_{3}=c_{4}=\ell_{1}$ and $c_{5}=0$. Material 2, laying above the interface, has a shear modulus $\mu_{2}$, and an internal length scale $\ell_{2}$ by taking $c_{1}=c_{2}=c_{3}=c_{4}=\ell_{2}$ and $c_{5}=0$.

For this bimaterial system, conventional elasticity theory dictates that the shear stress is uniform and the shear strain jumps in magnitude at the interface from $\varepsilon_{12}=\sigma_{12}^{\infty} / 2 \mu_{1}$ in material 1 to $\varepsilon_{12}=\sigma_{12}^{\infty} / 2 \mu_{2}$ in material 2 .

By including strain gradient effects, a continuously distributed shear strain can be obtained. In this problem, the only non-zero displacement, strain, stress and higher-order stress are $u_{1}, \varepsilon_{12}, \sigma_{12}$ and $\tau_{221}$, respectively, and they are functions of the co-ordinate $x_{2}$ only. From the 
constitutive equation (6), it follows that

$$
\sigma_{12}=2 \mu_{i} \varepsilon_{12} \quad \text { and } \quad \tau_{221}=2 \mu \ell_{i}^{2} \eta_{221}=4 \mu \ell_{i}^{2} \frac{\partial \varepsilon_{12}}{\partial x_{2}}
$$

in material $i$. Substitution of the above relations into the equilibrium equation (2) leads to

$$
\frac{\partial \varepsilon_{12}}{\partial x_{2}}-\hat{\ell}_{i}^{2} \frac{\partial^{3} \varepsilon_{12}}{\partial x_{2}^{3}}=0
$$

where $\hat{\ell}_{i}=\sqrt{2} \ell_{i}$. The general solution to the above ordinary differential equation is

$$
\varepsilon_{12}=d_{1}+d_{2} \mathrm{e}^{x_{2} / \hat{\ell}_{1}}+d_{3} \mathrm{e}^{-x_{2} / \hat{\ell}_{1}} \text { for } x_{2}<0
$$

and

$$
\varepsilon_{12}=d_{4}+d_{5} \mathrm{e}^{x_{2} / \hat{t}_{2}}+d_{6} \mathrm{e}^{-x_{2} / \hat{t}_{2}} \text { for } x_{2}>0
$$

Here $d_{1}$ to $d_{6}$ are 6 constants yet to be determined. The general solution is subject to the following boundary conditions:

(i) $\varepsilon_{12} \rightarrow \sigma_{12}^{\infty} / 2 \mu_{1}$ and $x_{2} \rightarrow-\infty$ and $\varepsilon_{12} \rightarrow \sigma_{12}^{\infty} / 2 \mu_{2}$ as $x_{2} \rightarrow \infty$

and at the interface;

(ii) continuity of traction: $\left.\left(\sigma_{21}-\tau_{221,2}\right)\right|_{x_{2} \rightarrow 0^{-}}=\left.\left(\sigma_{21}-\tau_{221,2}\right)\right|_{x_{2} \rightarrow 0^{+}}$;

(iii) continuity of double stress traction: $\left.\tau_{221}\right|_{x_{2} \rightarrow 0^{-}}=\left.\tau_{221}\right|_{x_{2} \rightarrow 0^{+}}$;

(iv) continuity of strain: $\left.\varepsilon_{12}\right|_{x_{2} \rightarrow 0^{-}}=\left.\varepsilon_{12}\right|_{x_{2} \rightarrow 0^{+}}$.

The particular solution satisfying all these conditions is

$$
\varepsilon_{12}=\frac{\sigma_{12}^{\infty}}{2 \mu_{1}}\left\{1+\frac{\mu_{1}-\mu_{2}}{\mu_{2}} \frac{\mu_{2} \hat{\ell}_{2}}{\mu_{1} \hat{\ell}_{1}+\mu_{2} \hat{\ell}_{2}} \mathrm{e}^{x_{2} / \hat{\ell}_{1}}\right\} \text { for } x_{2}<0
$$

and

$$
\varepsilon_{12}=\frac{\sigma_{12}^{\infty}}{2 \mu_{2}}\left\{1+\frac{\mu_{2}-\mu_{1}}{\mu_{1}} \frac{\mu_{1} \hat{\ell}_{1}}{\mu_{1} \hat{\ell}_{1}+\mu_{2} \hat{\ell}_{2}} \mathrm{e}^{-x_{2} / \hat{\ell}_{2}}\right\} \text { for } x_{2}>0
$$

\section{ACKNOWLEDGEMENTS}

This work is supported by the U.S. Department of Energy and the Lawrence Livermore National Laboratory under contract W-7405-Eng-48. The authors acknowledge many helpful comments by a reviewer.

\section{REFERENCES}

1. E. Cosserat and F. Cosserat, Theorie des Corps Deformables, Hermann et Fils, Paris, 1909.

2. R. A. Toupin, 'Elastic materials with couple stresses', Arch. Rational Mech. Anal., 11, 385-414 (1962).

3. R. D. Mindlin, 'Micro-structure in linear elasticity', Arch. Rational Mech. Anal., 16, 51-78 (1964).

4. E. C. Aifantis, 'On the microstructural origin of certain inelastic models', Trans. ASME J. Engng. Mater. Tech., 106, 326-330 (1984).

5. N. A. Fleck and J. W. Hutchinson, 'A phenomenological theory for strain gradient effects in plasticity', J. Mech. Phys. Solids, 41, 1825-1857 (1993).

6. N. A. Fleck and J. W. Hutchinson, 'Strain gradient plasticity', Adv. Appl. Mech., 33, 295-361 (1997). 
7. O. C. Zienkiewicz and R. L. Taylor, The Finite Element, 4th edn., McGraw-Hill, New York, 1994.

8. B. Specht, 'Modified shape functions for the three node plate bending element passing the patch test', Int. J. Numer. Meth. Engng., 26, 705-715 (1988).

9. Z. C. Xia and J. W. Hutchinson, 'Crack tip fields in strain gradient plasticity', J. Mech. Phys. Solids, 44, 1621-1648 (1996).

10. J. Y. Shu and N. A. Fleck, 'Prediction of a size effect in micro indentation', Int. J. Solids Struct., 35, 1363-1383 (1998).

11. L. R. Herrmann, 'Mixed finite elements for couple-stress analysis', in S. N. Atluri, R. H. Gallagher and O. C. Zienkiewicz (eds.), Hybrid and Mixed Finite Element Methods, Wiley, New York, 1983.

12. J. Y. Shu and N. A. Fleck, 'Strain gradient crystal plasticity: size-dependent deformation of a bicrystal', J. Mech. Phys. Solids (1998), in press.

13. V. P. Smyshlyaev and N. A. Fleck, 'The role of strain gradients in the grain size effect for polycrystals', J. Mech. Phys. Solids, 44, 465-495 (1996).

14. O. C. Zienkiewicz, J. P. Vilotte and S. Toyoshima, 'Iterative method for constrained and mixed approximation-an inexpensive improvement of f.e.m. performance', Comput. Meth. Appl. Mech. Engng., 51, 3-29 (1985).

15. M. E. Laursen and M. Gellert, 'Some criteria for numerical integrated matrices and quadrature formulas for triangles', Int. J. Numer. Meth. Engng., 12, 67-76 (1978).

16. R. D. Mindlin, 'Influence of couple-stresses on stress concentrations', Exp. Mech., 3, 1-7 (1963).

17. E. Hinton, F. C. Scott and R. E. Ricketts, 'Local least squares stress smoothing for parabolic isoparametric elements', Int. J. Numer. Meth. Engng., 9, 235-256 (1975). 